\title{
Novo Algoritmo para Atribuição de Potência por Circuito em Redes Ópticas Elásticas
}

\author{
Alexandre Fontinele ${ }^{1}$, Iallen Santos ${ }^{1,3}$, Jurandir Lacerda Jr. ${ }^{2,4}$, \\ André Soares $^{2}$, Adolfo V. T. Cartaxo ${ }^{5}$ e Divanilson R. Campelo ${ }^{1}$ \\ ${ }^{1}$ Centro de Informática (CIn) - Universidade Federal de Pernambuco (UFPE) \\ Recife - PE - Brasil \\ ${ }^{2}$ Departamento de Computação - Universidade Federal do Piauí (UFPI) \\ Teresina - PI - Brasil \\ ${ }^{3}$ Instituto Federal de Educação, Ciência e Tecnologia do Piauí (IFPI) \\ Piripiri - PI - Brasil \\ ${ }^{4}$ Instituto Federal de Educação, Ciência e Tecnologia do Piauí (IFPI) \\ Corrente - PI - Brasil \\ ${ }^{5}$ Instituto de Telecomunicações, Departamento de Ciências e Tecnologias da \\ Informação (DCTI), Instituto Universitário de Lisboa (ISCTE), Lisboa, Portugal \\ acf3ecin.upfe.br
}

\begin{abstract}
This paper presents a study on elastic optical networks that considers the power, routing, modulation level and spectrum assignment (PRMLSA) problem. The present study focuses on power assignment per circuit. In this context, the power assignment by binary search (PABS) algorithm, a new algorithm that performs adaptive power assignment per circuit, is proposed. The $P A B S$ algorithm is compared with other algorithms present in the literature in terms of bandwidth blocking probability. The PABS algorithm achieves a reduction of the bandwidth blocking probability that exceeds $39 \%$ in the NSFNet topology and $42 \%$ in the Cost 239 topology.
\end{abstract}

Resumo. Este artigo apresenta um estudo em redes ópticas elásticas considerando o problema de Power, Routing, Modulation Level and Spectrum Assignment (PRMLSA). O presente estudo se concentra na atribuição de potência por circuito. Nesse contexto, o algoritmo de Power Assignment by Binary Search (PABS), um novo algoritmo que realiza atribuição de potência por circuito de forma adaptativa, é proposto. O algoritmo PABS é comparado com outros algoritmos presentes na literatura em termos de probabilidade de bloqueio de banda. $O$ algoritmo PABS consegue uma redução na probabilidade de bloqueio de banda de pelo menos $39 \%$ na topologia NSFNet e $42 \%$ na topologia Cost 239 .

\section{Introdução}

As redes ópticas elásticas (EONs - Elastic Optical Networks) atraíram interesse de pesquisa nos últimos anos porque podem melhorar significativamente a eficiência espectral da camada óptica com alocação flexível de largura de banda. EONs permitem a divisão 
do espectro óptico em pequenas faixas de frequência chamadas slots. Assim, uma quantidade diferente de slots pode ser atribuída a cada requisição de circuito, de acordo com a demanda de largura de banda [Chatterjee et al. 2015].

A busca por uma rota, modulação e faixa de espectro para o estabelecimento de um circuito óptico corresponde ao problema de roteamento, seleção de modulação e alocação de espectro (RMLSA - Routing, Modulation Level, and Spectrum Assignment) [Christodoulopoulos et al. 2011]. O problema RMLSA consiste em: i) definir uma rota para um par de nós de origem e destino; $i i)$ selecionar uma modulação adequada para esta rota; e iii) escolher uma faixa de espectro livre (isto é, um conjunto de faixas contínuas e contíguas) na rota definida. Soluções para o problema RMLSA que levam em consideração a fragmentação do espectro, que é um problema que afeta severamente o desempenho das EONs, tendem a alcançar melhores desempenhos [Talebi et al. 2014, Liu et al. 2017].

Nas EONs transparentes, objeto de estudo deste trabalho, os dados são transmitidos da fonte ao destino como um sinal óptico. Toda a operação de roteamento é realizada no domínio óptico sem qualquer conversão no domínio eletrônico. A propagação do sinal ao longo de vários segmentos de fibra e elementos de amplificação resulta em acúmulo de ruído que pode impossibilitar que as soluções ao problema RMLSA atinjam qualidade de transmissão (QoT - Quality of Transmission) adequada [Beyranvand and Salehi 2013]. Esses ruídos são provenientes dos efeitos de camada física. Soluções RMLSA que contornam esses efeitos permitem uma redução da probabilidade de bloqueio [Beyranvand and Salehi 2013, Fontinele et al. 2017].

A potência atribuída para um circuito exerce influência sobre os efeitos de camada física. Os trabalhos publicados na literatura normalmente tratam o estabelecimento de circuitos em EONs com valores fixos para a potência ou densidade espectral de potência, enquanto outros aspectos como roteamento, modulação e espectro são selecionados de forma dinâmica [Christodoulopoulos et al. 2011, Yan et al. 2015, Moura et al. 2015]. Trabalhos mais recentes consideram a utilização de diferentes potências para cada circuito. Desta forma, o RMLSA é expandido para o problema Power, Routing, Modulation Level and Spectrum Assignment (PRMLSA) [Vale and Almeida 2019].

Neste artigo, é apresentado um novo algoritmo de atribuição de potência por circuito, chamado de Power Assignment by Binary Search (PABS). O algoritmo PABS busca atribuir potências de forma adaptativa no momento da requisição de um circuito. Foi realizado um estudo de avaliação de desempenho que comprovou a eficácia do PABS, em relação a outros algoritmos de atribuição de potência por circuito propostos na literatura, em termos de probabilidade de bloqueio de banda nas topologias NSFNet e Cost239.

As demais seções deste artigo estão organizadas da seguinte forma. A Seção 2 apresenta o problema de atribuição de potência por circuito e discute os trabalhos relacionados. O algoritmo proposto é apresentado na Seção 3. A Seção 4 apresenta a avaliação de desempenho dos algoritmos de atribuição de potência por circuito. Por fim, as conclusões do trabalho são apresentadas na Seção 5.

\section{Problema de Atribuição de Potência por Circuito}

Os efeitos da camada física degradam o sinal óptico quando ele se propaga ao longo do caminho óptico. Amplificadores ópticos (geralmente EDFA - Erbium Doped Fiber Ampli- 
fier) adicionam ruído de emissão espontânea amplificada (ASE - Amplified Spontaneous Emission) ao sinal. Além disso, a interferência não linear (NLI - Nonlinear Impairment) também é gerada durante a propagação do sinal na fibra óptica. Os NLIs fazem com que os circuitos causem interferência entre si circuito [Vale and Almeida 2019].

Neste artigo, o modelo de camada física apresentado em [Yan et al. 2015, Johannisson and Agrell 2014] é adotado para avaliar o impacto do ruído ASE e NLI na relação sinal-ruído óptico (OSNR - Optical Signal-to-Noise Ratio). O modelo assume formatos de modulação multi-símbolo multiplexados na polarização com detecção coerente e compensação de dispersão realizada pelo processamento de sinal digital no domínio elétrico no lado do receptor.

A potência de NLI depende das características do sinal (potência, largura de banda e posição no espectro). Por outro lado, a potência de ruído ASE depende da distância percorrida pelo sinal óptico e não é influenciada pelos níveis de potência de sinal. Entretanto, altos níveis de potência de sinal aumentam a potência de NLI na rede. Desta forma, a OSNR alcançada por um circuito depende, entre outros fatores, do nível de potência de sinal atribuída ao circuito [Vale and Almeida 2019].

Neste trabalho, a OSNR é usada como critério de QoT. Caso o valor da OSNR alcançada pelo circuito não esteja acima ou igual a um limiar de OSNR preestabelecido, o estabelecimento do circuito pode ser bloqueado por QoT inadequada. Um bloqueio por QoT inadequada pode acontecer de duas formas: $i$ ) QoT inadequada para o novo circuito (QoTN) ou ii) QoT inadequada para um ou mais circuitos já ativos na rede (QoTO). O QoTN é o bloqueio sofrido caso um novo circuito não atinja os níveis adequados de QoT. Mesmo que um novo circuito atinja tal requisito, ele ainda poderá sofrer bloqueio caso o estabelecimento do novo circuito torne inadequada a QoT de algum circuito já estabelecido, ocasionando assim o QoTO [Fontinele et al. 2017].

A Figura 1 apresenta exemplos de níveis de potência e as respectivas OSNRs alcançadas. Observa-se na Figura 1 que uma potência muito baixa $\left(P_{1}\right)$ vai gerar uma OSNR muita baixa $\left(O S N R_{1}\right)$ para o circuito. Um circuito que utilizar esse nível de potência será bloqueado devido a sua OSNR estar abaixo do limiar de OSNR $\left(O S N R_{t h}\right)$ exigido para o estabelecimento do circuito. O ideal é buscar um nível de potência entre a potência que alcança o limiar de OSNR e a potência que alcança a OSNR máxima $\left(O S N R_{\max }\right)$.

Os autores em [Palkopoulou et al. 2013] apresentam três estratégias para atribuir potência por circuito: Optimum Launch Power (OLP), Constant Launch Power (CLP) e Constant Power Spectral Density (CPSD). Os autores em [Vale and Almeida 2019] apresentam quatro estratégias para determinar a potência por circuito: Egoistic Power Assignment (EPA), Enough Power Assignment (EnPA), Constant Power Assignment (CPA) e Adaptive Power Assignment (APA). As estratégias OLP e CLP são semelhantes às estratégias EPA e CAP, respectivamente.

A estratégia EPA atribui ao circuito a potência que alcança o maior valor de OSNR $\left(O S N R_{\max }\right)$. Na estratégia EPA, a potência $\left(P_{\max }\right)$ que alcança a $O S N R_{\max }$ para um determinado circuito (ver Figura 1) é obtida usando a equação

$$
P_{\text {max }}=\frac{c_{\text {Route }}}{2 a_{\text {Channel }}}
$$




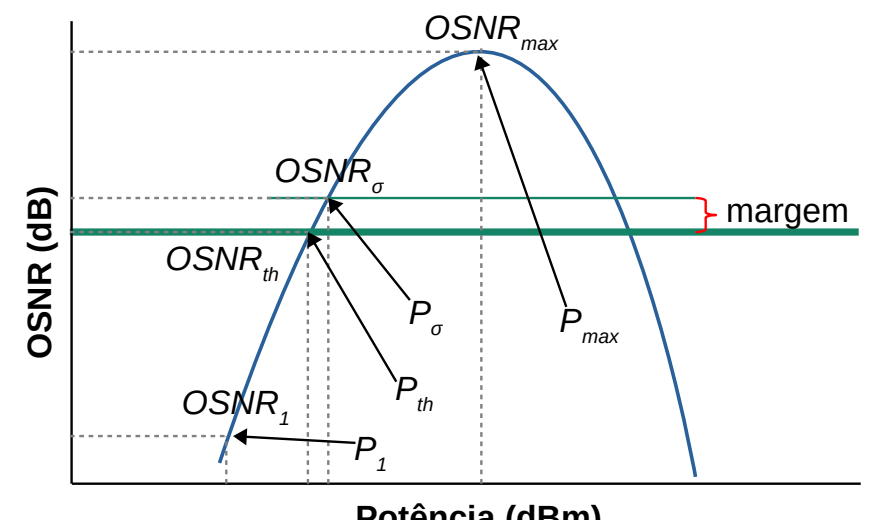

Potência (dBm)

Figura 1. Níveis de potência e suas respectivas OSNRs.

em que a forma de como obter os valores de $c_{\text {Route }}$ e $a_{\text {Channel }}$ pode ser encontrada em [Vale and Almeida 2019].

A estratégia EnPA atribui ao circuito a potência que alcança o limiar de OSNR $\left(O S N R_{t h}\right)$ da modulação selecionada para o circuito. Os autores em [Vale and Almeida 2019] utilizam interpolação linear entre uma potência muito baixa e a potência que alcança a OSNR máxima. Depois é realizado um ajuste para encontrar a potência que alcança a $O S N R_{t h}$. A Figura 1 apresenta um exemplo da potência $\left(P_{t h}\right)$ que alcança a $O S N R_{t h}$ e uma potência muito baixa $P_{1}$.

A estratégia CPA atribui a mesma potência para todos os circuitos. São analisados vários valores de potência, partindo de uma potência mínima até uma potência máxima para definir qual potência será atribuída aos circuitos. Aquele valor de potência que apresentar a menor probabilidade de bloqueio é o valor selecionado.

Na estratégia CPSD é utilizada a mesma densidade espectral de potência para todos os circuitos. A potência de cada circuito é proporcional à largura de banda alocada para o circuito. Para encontrar a densidade espectral de potência adequada são avaliados vários valores para a densidade espectral de potência, partindo de uma densidade menor para uma maior. Aquele valor que apresentar a menor taxa de bloqueio será selecionado.

O algoritmo APA atribui um valor de potência $\left(P_{\sigma}\right)$, correspondente ao limiar de OSNR adicionada de uma margem para prevenir interferências de futuros circuitos. Por esta razão, o valor de potência $P_{\sigma}$ deverá ser maior do que a potência $P_{t h}$ e menor do que a potência $P_{\max }$ (ver Figura 1). Potências já utilizadas para o estabelecimento de circuitos são salvas em um banco de dados. Quando o atendimento de uma requisição de circuito que não possui um valor de potência salvo no banco de dados, o valor inicial de potência $\left(P_{\text {Current }}\right)$ é obtido usando a equação:

$$
P_{\text {Current }}=P_{\min }+k_{p} \times\left(P_{\max }-P_{\min }\right),
$$

em que o parâmetro $k_{p}$ é responsável por ajustar a margem de potência relativa à potência de limiar, $P_{t h}$. $P_{\max }$ pode ser inicializada com a Equação 1 e a estratégia EnPA pode ser usar para inicializar $P_{\text {min }}$. Se a OSNR $\left(O S N R_{\text {Current }}\right)$ alcançada com $P_{\text {Current }}$ for maior ou igual a $O S N R_{t h}$ e a QoT de algum circuito já ativo na rede não se tornar inadequada com o estabelecimento do novo circuito, o algoritmo APA salva a nova potência 
no banco de dados e termina sua execução. No caso da $O S N R_{\text {Current }}$ for maior ou igual a $O S N R_{t h}$, mas algum circuito ficar com a sua QoT inadequada com o estabelecimento do novo circuito, o algoritmo APA aplica a equação:

$$
P_{\text {Current }}^{\prime}=P_{\text {Current }}-0,1 \times P_{\text {Current }},
$$

para ajustar a potência $P_{\text {Current }}$ para uma nova potência $P_{\text {Current }}^{\prime}$. Depois de calcular a nova potência, o algoritmo APA realiza outra vez as verificações de $O S N R_{t h}$ e QoT dos outros circuitos. Se a OSNR $\left(O S N R_{\text {Current }}\right)$ ficar menor que $O S N R_{t h}$ o algoritmo APA aplica a equação:

$$
P_{\text {Current }}^{\prime}=P_{\text {Current }}+0,1 \times P_{\text {Current }},
$$

para o ajuste da potência $P_{\text {Current }}$ para uma nova potência $P_{C u r r e n t}^{\prime}$ e depois realiza as verificações de $O S N R_{t h}$ e QoT dos outros circuitos. O algoritmo APA possui contadores para contabilizar o número de vezes que as equações 3 e 4 são aplicadas. Esses contadores impedem que o algoritmo APA entre em um loop infinito de adições e subtrações.

\section{Algoritmo Proposto}

O algoritmo proposto baseia-se no método de busca binária para atribuição de potência por circuito de forma adaptativa. O algoritmo é chamado de Power Assignment by Binary Search (PABS). O algoritmo PABS adota a estratégia de usar um banco de dados que guarda as potências atribuídas para circuitos já estabelecidos na rede. Associada a cada potência salva no banco de dados, são salvadas também as seguintes informações: rota, taxa de transmissão e modulação. O Algoritmo 1 apresenta os passos do PABS para encontrar a potência que será atribuída para um novo circuito.

O parâmetros utilizados pelo Algoritmo 1 são requisição de circuito $(C)$, rota $(R)$, modulação $(M)$, faixa de espectro livre $(S)$ e margem de OSNR desejada (OSNR $\left.R_{\text {margin }}\right)$. A requisição de circuito contém informações como origem, destino e taxa de transmissão. A margem de OSNR desejada é um parâmetro ajustável que o algoritmo utiliza na busca do melhor valor para a potência quer será atribuída para o novo circuito. Um valor zero para a margem significa que será procurada uma potência mínima que alcance o limiar de OSNR da modulação selecionada para o novo circuito. Valores maiores que zero para a margem fazem com que o algoritmo procure por potências com valores acima da potência mínima mencionada anteriormente. Atribuir valores maiores que zero para a margem mostrou-se uma boa estratégia para lidar com interferências futuras de outros circuitos [Vale and Almeida 2019, Fontinele et al. 2017].

A expressão que o algoritmo PABS utiliza para buscar por uma potência para um novo circuito está na linha 18 do Algoritmo 1. Essa expressão também é utilizada para ajustar uma potência já existente no banco de dados para o estado atual da rede. O valor de $P_{\max }$ é calculado na linha 8 do Algoritmo 1 e utiliza a Equação 1. Já o valor de $P_{\text {min }}$ é inicializado com uma potência bem baixa, foi utilizada $-80 \mathrm{dBm}$ para os estudos deste trabalho. No algoritmo APA, a $P_{\min }$ é inicializada com um valor de potência que corresponde à potência mínima que alcança o valor do limiar de OSNR.

A variável $P_{\text {Current }}$ guarda o valor da potência encontrado até ao momento para o novo circuito. Usam-se dois critérios para saber se a potência em $P_{\text {Current }}$ deve ser 


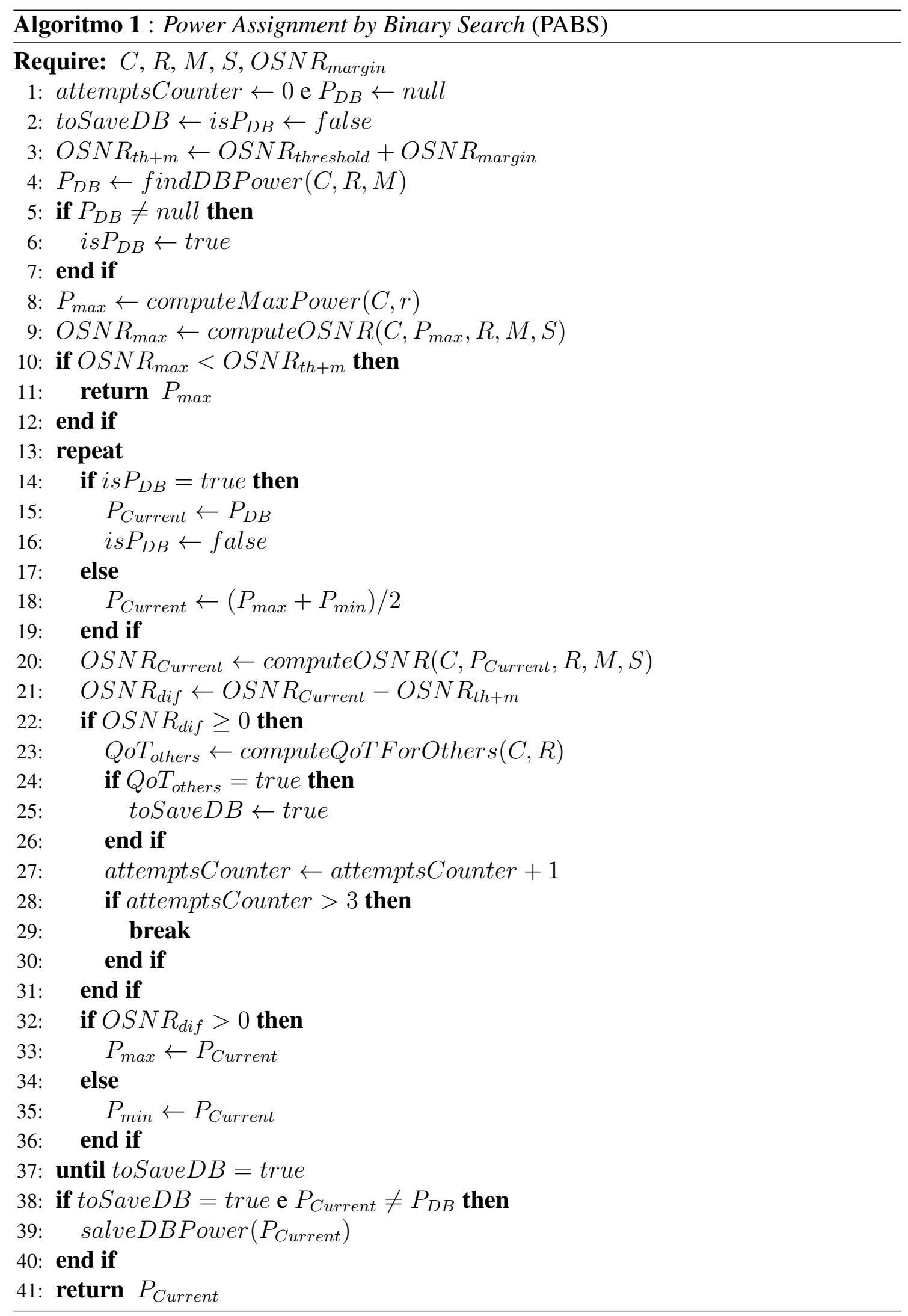

usada pelo novo circuito para o seu estabelecimento: $i$ ) a OSNR alcançada pela potência $P_{\text {Current }}$ deve ser maior ou igual a $O S N R_{t h+m}$ e $\left.i i\right)$ não tornar inadequada a QoT de 
algum circuito já ativo na rede. A verificação dos critérios é realizada nas linhas 20, 21, 22, 23 e 24 do Algoritmo 1. Se os dois critérios forem atendidos, a nova potência pode ser salva no banco de dados e será retornada para ser usada pelo novo circuito. Pode acontecer que a potência encontrada não consiga satisfazer os dois critérios. $\mathrm{O}$ algoritmo verifica apenas três vezes os dois critérios para evitar que entre em loop infinito. Ele sempre retorna uma potência, mesmo que ela não satisfaça os dois critérios. O algoritmo PRMLSA que utilizar o PABS ficará encarregado de avaliar se a potência retornada gera o bloqueio do novo circuito.

O algoritmo PABS, assim como os outros algoritmos de atribuição de potência por circuito, é aplicado em conjunto com os algoritmos RMLSA [Vale and Almeida 2019]. É importante destacar que o plano de controle compreende um conjunto de protocolos responsáveis pelo provisionamento dinâmico de circuitos [Sócrates-Dantas et al. 2014]. No caso deste trabalho, o plano de controle é responsável pela aplicação dos algoritmos PRMLSA. Ele também armazena informações sobre a arquitetura de rede e seu estado atual. O Algoritmo 2 apresenta o algoritmo PRMLSA integrado que foi adotado para a realização dos estudos deste trabalho.

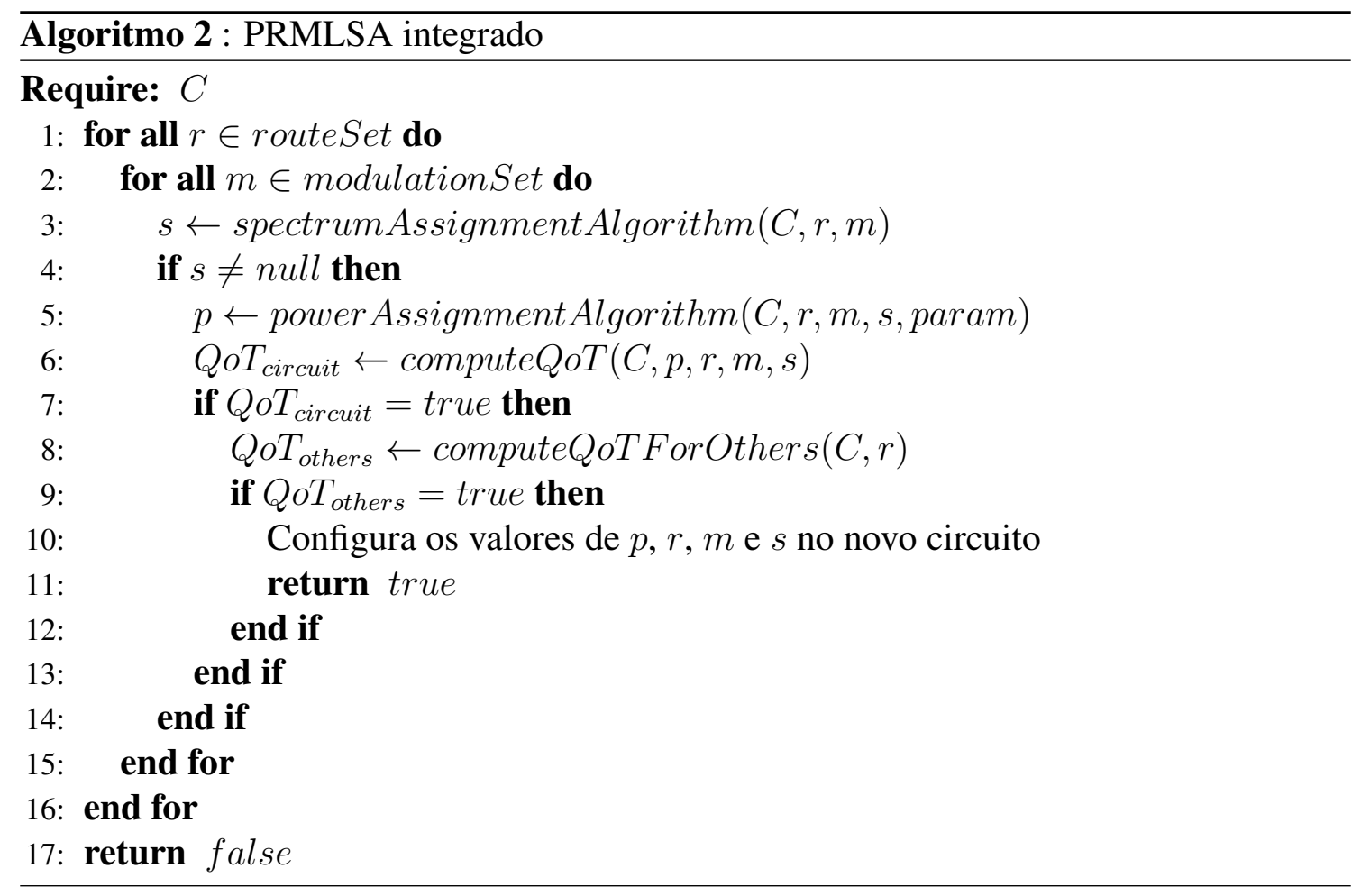

$\mathrm{Na}$ aplicação do Algoritmo 2 as rotas estão ordenadas em ordem crescente de distância e as modulações estão ordenadas em ordem decrescente de eficiência espectral. O algoritmo de atribuição de potência selecionado é aplicado na linha 5 do Algoritmo 2. O Algoritmo 2 verifica a QoT do novo circuito e as QoTs dos demais circuitos já ativos na rede nas linhas 6, 7, 8 e 9. Dessa forma, o algoritmo PRMLSA integrado pode identificar se o algoritmo de atribuição de potência retornou uma potência que leva ao bloqueio da requisição. Caso isso ocorra, o algoritmo PRMLSA integrado vai seguir para a próxima etapa e tentar solicitar uma potência adequada. O plano de controle fica encarregado de identificar o tipo de bloqueio que a requisição sofreu no caso do Algoritmo 2 retornar 
false. Caso contrário, o plano de controle estabelecerá a requisição.

\section{Avaliação de Desempenho}

Esta seção apresenta a avaliação de desempenho dos seguintes algoritmos de atribuição de potência por circuito: EPA, EnPA, CPA, CPSD, APA e PABS. O objetivo deste estudo de avaliação de desempenho é demonstrar a eficácia do algoritmo proposto em relação às demais propostas para atribuição de potência encontradas na literatura. Para avaliar os algoritmos de atribuição de potência em diferentes cenários, o estudo de avaliação de desempenho também considera os seguintes fatores: topologias de rede (NSFNet e Cost239) e carga na rede.

As topologias NSFNet (National Science Foundation Network) e Cost239 são apresentadas na Figura 2 [Zhao et al. 2018]. A topologia NSFNet tem 14 nós com conectividade média de 3,14 enlaces por nó e 22 enlaces com comprimento médio de $918,18 \mathrm{~km}$. A topologia Cost 239 tem 11 nós com conectividade média de 4,73 enlaces por nó e 26 enlaces com comprimento médio de 786,35km.

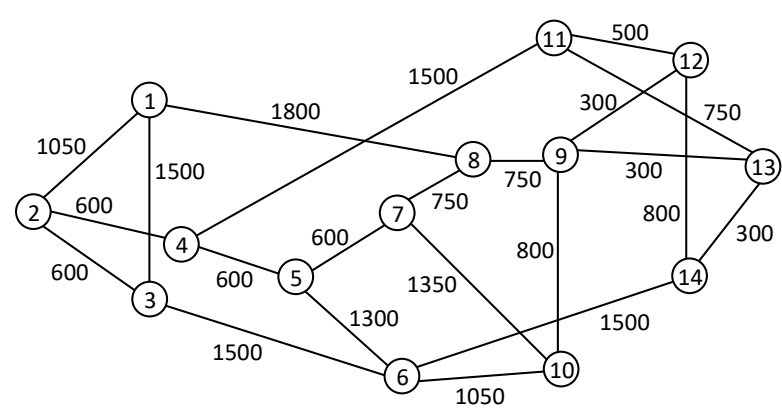

(a)

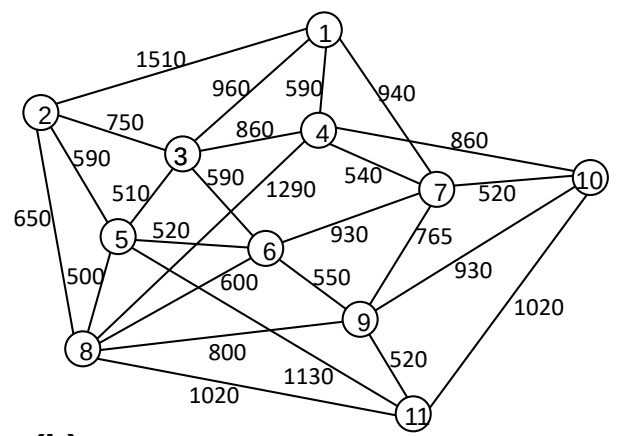

(b)

\section{Figura 2. Topologias consideradas no estudo: (a) NSFNet e (b) Cost239. 0 número em cada enlace corresponde a distância em km.}

Como este trabalho é focado nos algoritmos de atribuição de potência, não foram adotados algoritmos muito sofisticados (soluções mais complexas apresentadas pela literatura) para a resolução do problema RMLSA (ver Algoritmo 2). Foi utilizado o algoritmo de $k$ menores caminhos para o roteamento, com $k$ igual a 3, a estratégia FirstFit para a alocação de espectro e a seleção da modulação é feita pela QoT.

A probabilidade de bloqueio de banda foi considerada como métrica de avaliação de desempenho. A probabilidade de bloqueio de banda é a razão entre a largura de banda bloqueada pela largura de banda total requisitada [Ruan and Zheng 2014]. Bloqueios de banda ocorrem por Fragmentação, QoTN (QoT inaceitável para um novo circuito), QoTO (QoT inaceitável para algum circuito já ativo na rede) e AEL (ausência de espectro livre).

Para avaliar o desempenho dos diferentes algoritmos, foram realizadas simulações com a ferramenta SNetS (SLICE Network Simulator) [Fontinele et al. 2017]. Em cada simulação, foram observadas 100.000 requisições de circuitos ópticos com quatro níveis de granularidade: 150, 200, 250 e $300 \mathrm{Gbps}$, com as proporções 4:3:2:1, respectivamente. A geração de requisições segue a distribuição de Poisson com a carga de tráfego distribuída uniformemente entre todos os pares de nós (origem e destino). Para cada simulação, foram realizadas dez replicações com diferentes sementes de geração de 
variável aleatória. Todos os resultados são apresentados com estimativa pontual e estimativa intervalar considerando o nível de confiança de $95 \%$.

As modulações consideradas neste estudo foram BPSK, QPSK, 8QAM, 16QAM e 32QAM e os seus respectivos limiares de SNR (Signal-to-Noise Ratio) por símbolo são 5,5, 8,5, 12,5, 15,1, 18,1 dB [Ives et al. 2015]. Os limiares de SNR por símbolo para as modulações foram calculados para atingir um pré FEC-BER $4 \cdot 10^{-3}$ e incluem um overhead de $12 \%$ para o FEC [Ives et al. 2015]. Vale ressaltar que os valores de limiar de OSNR são iguais aos valores de limiar de SNR por símbolo para o modelo de camada física adotado.

Todos os enlaces da rede são bidirecionais e possuem largura de banda do espectro dividida em 320 slots de frequência. Um slot de frequência possui largura de banda de 12,5 GHz. Foi utilizada a banda de guarda igual a um slot de frequência. Os ganhos dos amplificadores são ajustados para compensar as perdas dos dispositivos ópticos (comutador, multiplexador e demultiplexador) e das fibras. Outros parâmetros utilizados nas simulações estão listados na Tabela 1.

Tabela 1. Parâmetros de camada física utilizados nas simulações.

\begin{tabular}{c|c}
\hline Descrição & Valor \\
\hline Atenuação da fibra $(\alpha)$ & $0,2 \mathrm{~dB} / \mathrm{km}$ \\
Parâmetro de dispersão da fibra $(D)$ & $16 \mathrm{ps} /(\mathrm{nm} \cdot \mathrm{km})$ \\
Coeficiente não linear da fibra $(\gamma)$ & $1,3(\mathrm{~W} \cdot \mathrm{km})^{-1}$ \\
Tamanho de um span $\left(L_{s}\right)$ & $80 \mathrm{~km}$ \\
Figura de ruído do amplificador $(N F)$ & $5 \mathrm{~dB}$ \\
\hline
\end{tabular}

\subsection{Ajuste dos Parâmetros dos Algoritmos CPA, CPSD, APA e PABS}

Os algoritmos CPA, CPSD, APA e PABS possuem parâmetros ajustáveis que interferem diretamente nos seus desempenhos. Inicialmente, foram realizados experimentos para determinar os valores destes parâmetros buscando o melhor desempenho de cada algoritmo. Os experimentos consistiram em realizar simulações com valores predefinidos para os parâmetros dos algoritmos. Os valores partiam de um valor mínimo para um valor máximo com um certo nível de incremento. Depois foram analisadas as probabilidades de bloqueio de banda alcançadas com cada valor e foi selecionado o valor que alcançou a menor probabilidade de bloqueio de banda. Quando mais de um valor alcançou a menor probabilidade de bloqueio de banda considerando as estimativas intervalares, foi selecionado o valor com a menor estimativa pontual.

As Figuras 3 e 4 apresentam os resultados dos experimentos realizados com os algoritmos CPA, CPSD, APA e PABS. A carga na rede utilizada para ajustar os parâmetros dos algoritmos CPA, CPSD, APA e PABS corresponde à carga mais alta analisada em cada topologia (carga de 600 Elangs para a topologia NSFNet e carga de 1550 Erlangs para a topologia Cost239). Essa estratégia foi adotada considerando que os parâmetros que definem o desempenho dos algoritmos devem garantir que a rede suporte a capacidade da demanda planejada na implementação inicial e seja escalável durante toda a vida útil da rede, o que pode abranger várias décadas [Pointurier 2017].

Para o algoritmo CPA foi realizado um estudo utilizando a menor potência igual a $-10 \mathrm{dBm}$ e a maior igual a $0 \mathrm{dBm}$, com incremento de $1 \mathrm{~dB}$, Figura 3(a). As potências -3 


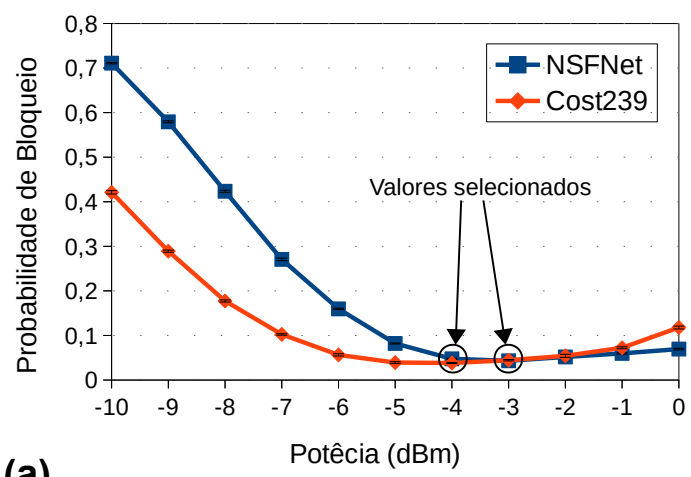

(a)

Figura 3. Probabilidade de bloqueio de banda obtida pelos algoritmos (a) CPA e (b) CPSD em função da potência.
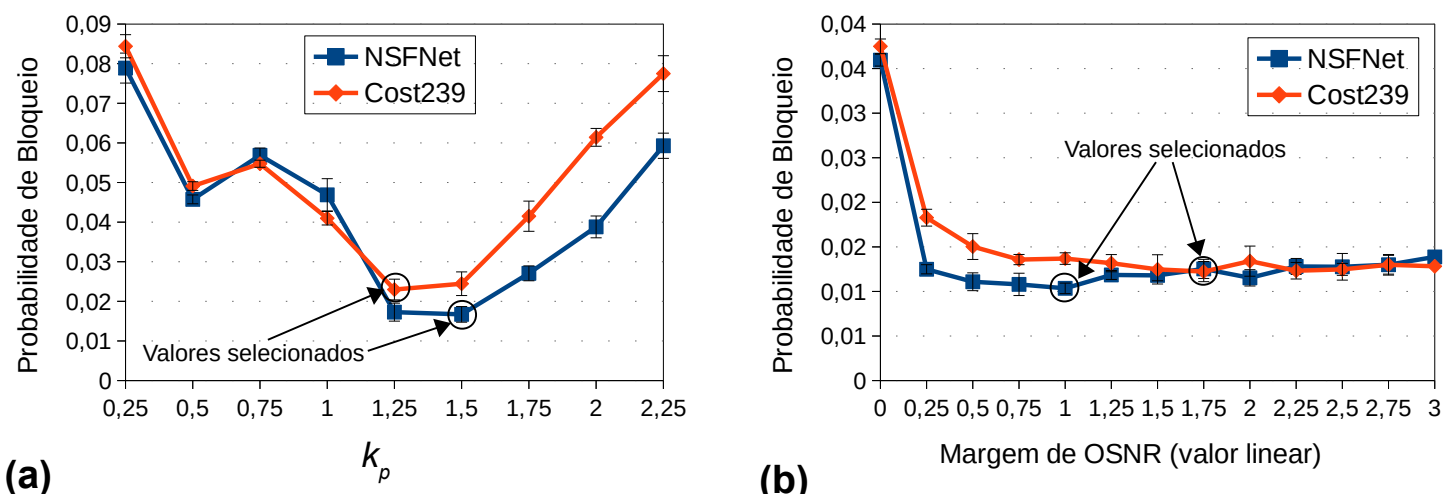

(b)

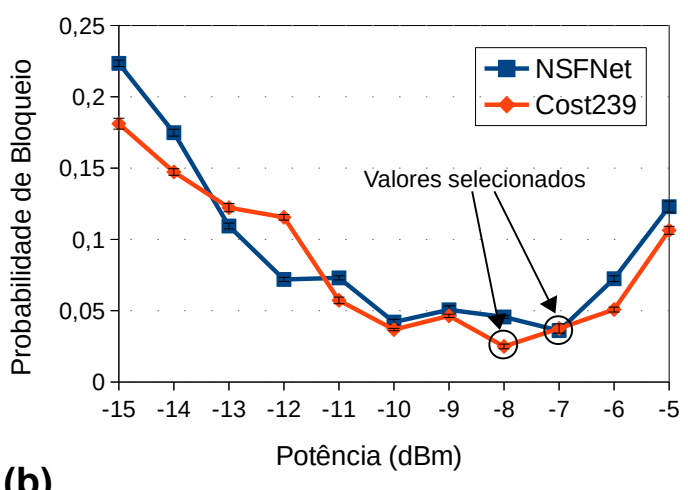

(b)

Figura 4. Probabilidade de bloqueio de banda obtida pelos algoritmos (a) APA e (b) PABS em função do parâmetro ajustável de cada algoritmo.

dBm e -4 dBm alcançaram os melhores desempenhos nas topologias NSFNet e Cost239, respectivamente. Para o algoritmo CPSD foi utilizada a menor potência igual a $-15 \mathrm{dBm}$ e a maior potência igual a $-5 \mathrm{dBm}$, com incremento de $1 \mathrm{~dB}$, Figura 3(b). Para encontrar a densidade espectral de potência foi utilizada a largura de banda de referência igual à largura de banda de um slot. As potências $-7 \mathrm{dBm}$ e $-8 \mathrm{dBm}$ alcançaram os melhores desempenhos nas topologias NSFNet e Cost239, respectivamente.

Para encontrar o melhor valor para o parâmetro usado pelo algoritmo APA foi avaliado o desempenho em que o parâmetro $k_{p}$ (equação 2) tomou valores entre 0,25 e 2,25, com incremento de 0,25, Figura 4(a). Os valores 1,5 e 1,25 apresentaram os melhores desempenhos nas topologias NSFNet e Cost239, respectivamente. Para o algoritmo PABS foi realizado um estudo para encontrar o valor da margem de OSNR que gera a menor probabilidade de bloqueio de banda. O menor valor utilizado foi 0 e o maior foi 3 , com incremento de 0,25, Figura 4(b). Os valores para a margem de OSNR que alcançaram os melhores desempenhos foram 1,0 e 1,75 nas topologias NSFNet e Cost239, respectivamente.

\subsection{Comparação entre os Algoritmos}

As Figuras 5 e 6 apresentam as probabilidades de bloqueio de banda obtidas pelos algoritmos de atribuição de potência por circuito em função da carga na rede para as topologias 
NSFNet e Cost239, respectivamente.

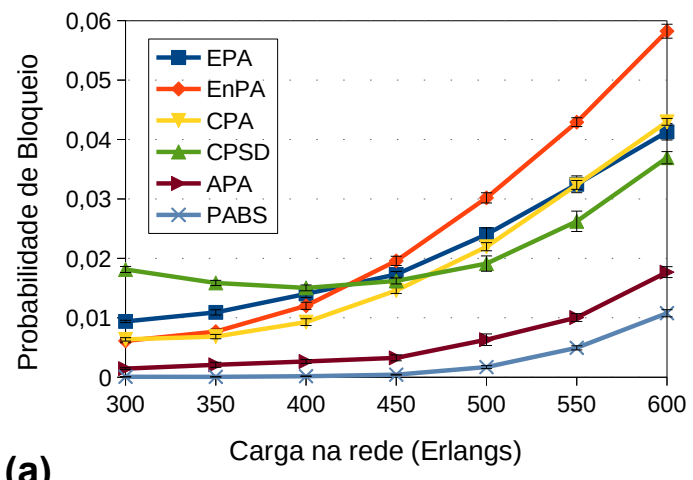

(a)

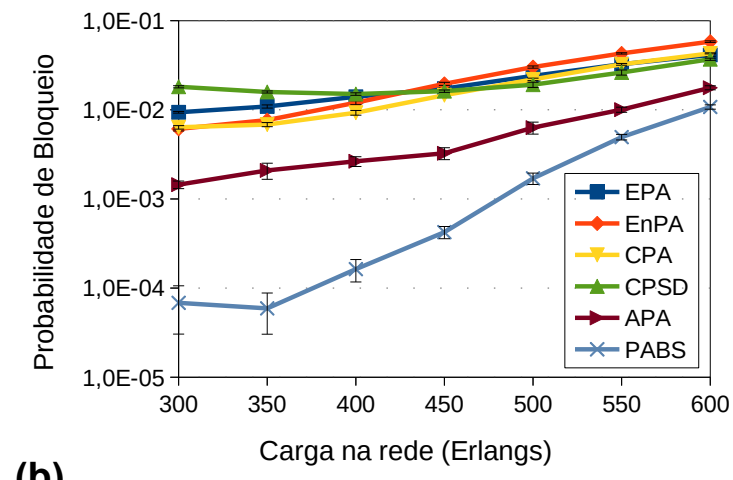

(b)

Figura 5. Probabilidade de bloqueio de banda obtida pelos algoritmos de atribuição de potência por circuito para a topologia NSFNet. (a) Escala normal e (b) Escala logarítmica.

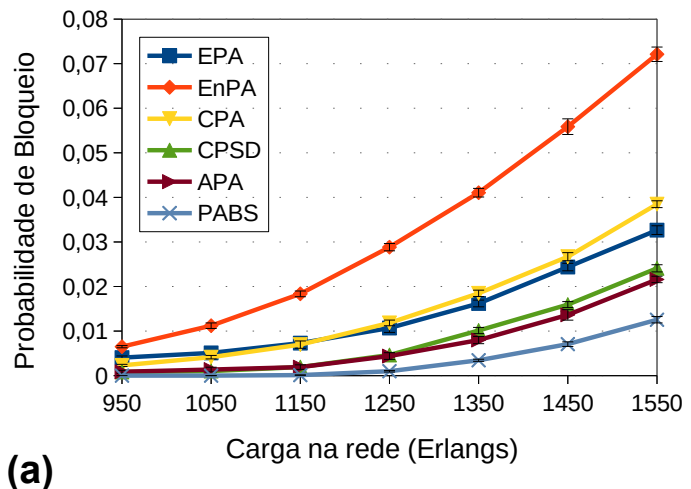

(a)

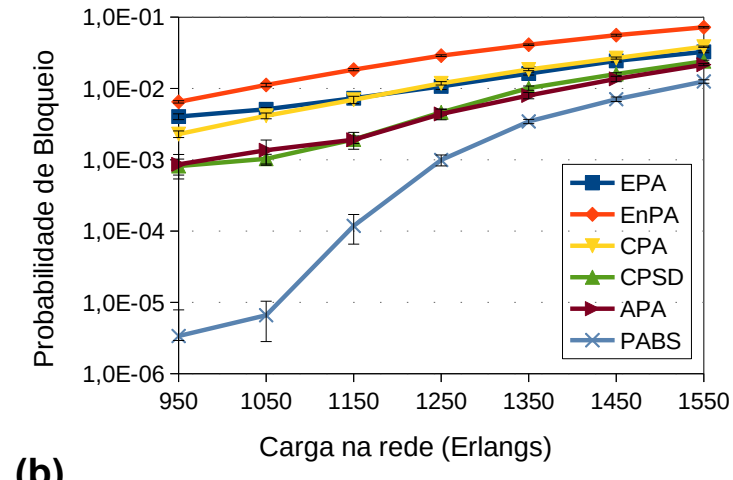

(b)

Figura 6. Probabilidade de bloqueio de banda obtida pelos algoritmos de atribuição de potência por circuito para a topologia Cost239. (a) Escala normal e (b) Escala logarítmica.

Nota-se pelas Figuras 5 e 6 que o algoritmo PABS alcançou o melhor desempenho em termos de probabilidade de bloqueio de banda em todos os cenários avaliados. $\mathrm{O}$ algoritmo APA apresentou o segundo melhor desempenho na topologia NSFNet. Na topologia Cost239 o desempenho do APA ficou semelhante ao CPSD. O algoritmo EnPA apresentou o pior desempenho em relação aos outros algoritmos na maioria das cargas analisadas nas duas topologias.

A Tabela 2 mostra a redução na probabilidade de bloqueio de banda alcançada pelo algoritmo PABS em relação aos demais algoritmos de atribuição de potência por circuito. Os valores de redução na probabilidade de bloqueio de banda exibidos na Tabela 2 correspondem ao menor valor obtido para todas as cargas testadas em ambas as topologias. Isto significa que em alguns pontos de carga o PABS apresenta redução de probabilidade de bloqueio de banda ainda maior (apresentou uma redução máxima de $100 \%$ em ambas topologias). Observa-se pela Tabela 2 que o algoritmo PABS alcança uma redução na probabilidade de bloqueio de banda de pelo menos $39 \%$ na topologia NSFNet e $42 \%$ na topologia Cost 239. 
Tabela 2. Redução mínima na probabilidade de bloqueio de banda alcançada pelo algoritmo PABS em relação a probabilidade de bloqueio de banda dos outros algoritmos de atribuição de potência por circuito.

\begin{tabular}{c|ccccc}
\hline Topologia & EPA & EnPA & CPA & CPSD & APA \\
\hline NSFNet & $74 \%$ & $82 \%$ & $75 \%$ & $71 \%$ & $39 \%$ \\
\hline Cost 239 & $62 \%$ & $83 \%$ & $67 \%$ & $48 \%$ & $42 \%$ \\
\hline
\end{tabular}

A Figura 7 apresenta a composição da probabilidade de bloqueio de banda obtida pelos algoritmos de atribuição de potência por circuito para as duas topologias consideradas nos estudos. A Figura 7(a) apresenta os resultados obtidos para a carga de 600 Erlangs na topologia NSFNet e a Figura 7(b) apresenta os resultados obtidos para a carga de 1550 Erlangs na topologia Cost239.
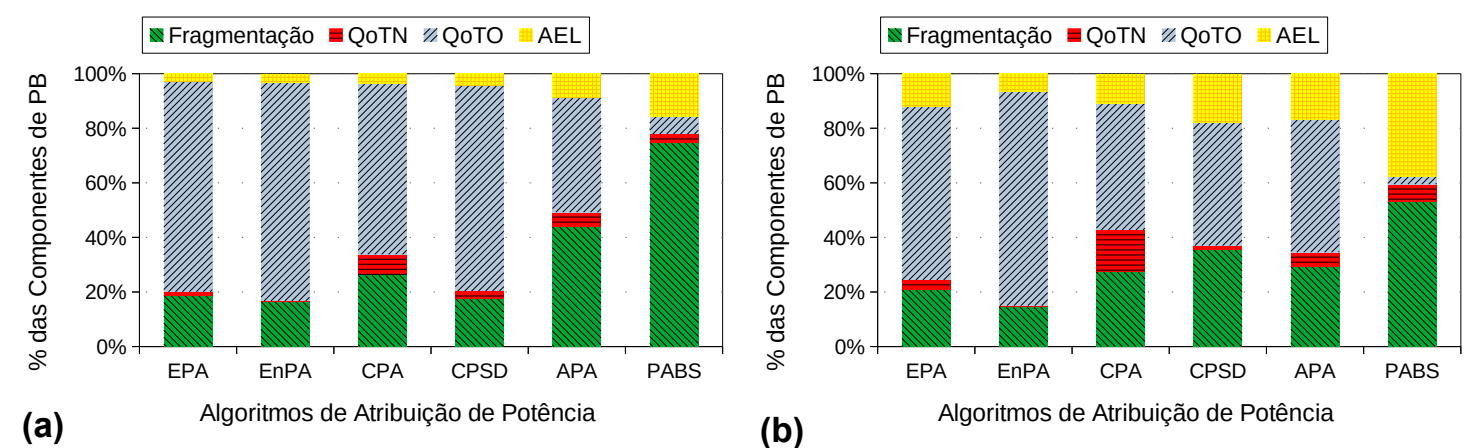

Figura 7. Percentagens das componentes da probabilidade de bloqueio de banda (PB) obtidas pelos algoritmos de atribuição de potência por circuito para as topologias (a) NSFNet e (b) Cost239.

Analisando a Figura 7, nota-se qual componente da probabilidade de bloqueio de banda impacta mais o desempenho dos algoritmos. $\mathrm{O}$ algoritmo EnPA busca atribuir potências que alcançam um valor de OSNR um pouco acima do limiar de OSNR da modulação selecionada para o circuito. Essa estratégia estabelece circuitos frágeis a interferências de outros circuitos. Observa-se pela Figura 7 que a componente que mais afetou o desempenho do algoritmo EnPA foi a QoTO. A componente QoTO está relacionada com os bloqueios gerados por interferências entre os circuitos.

A estratégia usada pelo algoritmo EPA é atribuir a potência que alcança a OSNR máxima por circuito. Essa estratégia pode levar ao estabelecimento de circuitos com níveis excessivos de potências. Isso leva a níveis altos de potências interferindo em outros circuitos, isto é, maior número de bloqueios por QoTO, Figura 7.

O algoritmo CPA, por atribuir o mesmo nível de potência para todos os circuitos, estabelece alguns circuitos frágeis e outros com excesso de potência. Isto também contribui para que a maioria dos bloqueios ocorridos com o uso do CPA sejam do tipo QoTO, Figura 7.

A estratégia de atribuir a mesma densidade espectral de potência para todos os circuitos, adotada pelo algoritmo CPSD, permite que a potência atribuída a cada circuito seja ajustada pela sua largura de banda. Essa estratégia possibilitou o melhor desempenho do algoritmo CPSD em relação aos algoritmos EPA, EnPA e CPA nas cargas mais altas 
na topologia NSFNet (Figura 5) e em todas as cargas na topologia Cost239 (Figura 6). Mesmo assim, o algoritmo CPSD ainda sofreu mais bloqueios do tipo QoTO, Figura 7.

Os algoritmos APA e PABS buscam atribuir valores de potência por circuito de forma adaptativa, entre valores mínimo e máximo. A cada novo ajuste na potência, os algoritmos APA e PABS realizam uma verificação do impacto de interferência que a nova potência causa nos outros circuitos já estabelecidos na rede. Dessa forma, eles conseguem superar as limitações dos algoritmos EPA, EnPA e CPA. Entretanto, o PABS se saiu melhor que o APA. A maioria dos bloqueios sofridos pelo PABS foram relacionados com os recursos espectrais (fragmentação e AEL), Figura 7.

A topologia NSFNet possui menor conectividade média dos nós e maior comprimento médio dos enlaces em relação a topologia Cost239. Essas características da topologia NSFNet contribuem para que a probabilidade de bloqueio devido às penalidades de camada física se acentuem nela. Isso fica evidenciado quando observa-se as menores reduções na probabilidade de bloqueio de banda alcançadas pelo algoritmo PABS em relação aos algoritmos EPA, CPA e CPSD nas duas topologias, Tabela 2.

\section{Conclusão}

Este artigo propõe um novo algoritmo para atribuição de potência por circuito em redes ópticas elásticas chamado de PABS. O algoritmo PABS realiza a atribuição de potência por circuito de forma adaptativa baseando-se no método de busca binária. $\mathrm{O}$ algoritmo PABS também utiliza um banco de dados para guardar as potências que foram utilizadas para o estabelecimento de circuitos. As potências salvas no banco de dados são utilizadas para evitar uma busca cega por novas potências para requisições de circuitos com característica semelhantes aos circuitos já estabelecidos.

Foi conduzida uma avaliação de desempenho comparando o algoritmo PABS com os algoritmos EPA, EnPA, CPA, CPSD e APA nas topologias NSFNet e Cost239. Os resultados mostraram que o algoritmo proposto alcançou o melhor desempenho em termos de probabilidade de bloqueio de banda em comparação aos outros algoritmos para todas as cargas analisadas em ambas as topologias. O algoritmo PABS obteve uma redução na probabilidade de bloqueio de banda em relação aos outros algoritmos que excede $39 \%$ na topologia NSFNet e que excede $42 \%$ na topologia Cost 239 .

Como trabalho futuro pretende-se avaliar o desempenho de algoritmos de atribuição de potência por circuito com algoritmos RMLSA sofisticados (por exemplo, um algoritmo de alocação de espectro que realiza particionamento do espectro). Pretende-se também conduzir estudos considerando o problema PRMLSA em redes ópticas elásticas translúcidas.

\section{Referências}

Beyranvand, H. and Salehi, J. (2013). A quality-of-transmission aware dynamic routing and spectrum assignment scheme for future elastic optical networks. IEEE/OSA Journal of Lightwave Technology, 31(18):3043-3054.

Chatterjee, B. C., Sarma, N., and Oki, E. (2015). Routing and spectrum allocation in elastic optical networks: A tutorial. IEEE Communications Surveys Tutorials, 17(3):17761800 . 
Christodoulopoulos, K., Tomkos, I., and Varvarigos, E. (2011). Elastic bandwidth allocation in flexible OFDM-based optical networks. IEEE/OSA Journal of Lightwave Technology, 29(9):1354-1366.

Fontinele, A., Santos, I., Neto, J. N., Campelo, D. R., and Soares, A. (2017). An efficient IA-RMLSA algorithm for transparent elastic optical networks. Computer Networks, 118(Supplement C): 1 - 14.

Ives, D. J., Bayvel, P., and Savory, S. J. (2015). Routing, modulation, spectrum and launch power assignment to maximize the traffic throughput of a nonlinear optical mesh network. Photonic Network Communications, 29(3):244-256.

Johannisson, P. and Agrell, E. (2014). Modeling of nonlinear signal distortion in fiberoptic networks. IEEE/OSA Journal of Lightwave Technology, 32(23):4544-4552.

Liu, H., Lv, L., Chen, Y., and Wei, C. (2017). Fragmentation-avoiding spectrum assignment strategy based on spectrum partition for elastic optical networks. IEEE Photonics Journal, 9(5):1-13.

Moura, P. M., Scaraficci, R. A., and d. Fonseca, N. L. S. (2015). Algorithm for energy efficient routing, modulation and spectrum assignment. In 2015 IEEE International Conference on Communications (ICC), pages 5961-5966.

Palkopoulou, E., Bosco, G., Carena, A., Klonidis, D., Poggiolini, P., and Tomkos, I. (2013). Nyquist-wdm-based flexible optical networks: exploring physical layer design parameters. IEEE/OSA Journal of Lightwave Technology, 31(14):2332-2339.

Pointurier, Y. (2017). Design of low-margin optical networks. IEEE/OSA Journal of Optical Communications and Networking, 9(1):A9-A17.

Ruan, L. and Zheng, Y. (2014). Dynamic survivable multipath routing and spectrum allocation in ofdm-based flexible optical networks. IEEE/OSA Journal of Optical Communications and Networking, 6(1):77-85.

Sócrates-Dantas, J., Careglio, D., Perelló, J., Silveira, R. M., Ruggiero, W. V., and SolèPareta, J. (2014). Challenges and requirements of a control plane for elastic optical networks. Computer Networks, 72:156 - 171.

Talebi, S., Alam, F., Katib, I., Khamis, M., Salama, R., and Rouskas, G. N. (2014). Spectrum management techniques for elastic optical networks: A survey. Optical Switching and Networking, 13:34-48.

Vale, V. and Almeida, R. (2019). Power, routing, modulation level and spectrum assignment in all-optical and elastic networks. Optical Switching and Networking, 32:14 24.

Yan, L., Agrell, E., Wymeersch, H., Johannisson, P., Di Taranto, R., and Brandt-Pearce, M. (2015). Link-level resource allocation for flexible-grid nonlinear fiber-optic communication systems. IEEE Photonics Technology Letters, 27(12):1250-1253.

Zhao, Y., Hu, L., Zhu, R., Yu, X., Wang, X., and Zhang, J. (2018). Crosstalk-aware spectrum defragmentation based on spectrum compactness in space division multiplexing enabled elastic optical networks with multicore fiber. IEEE Access, 6:15346-15355. 